\title{
GRB 050904 at redshift 6.3: observations of the oldest cosmic explosion after the Big Bang ${ }^{\star}$
}

\author{
G. Tagliaferri ${ }^{1}$, L. A. Antonelli ${ }^{2}$, G. Chincarini ${ }^{1,3}$, A. Fernández-Soto ${ }^{4}$, D. Malesani ${ }^{5}$, M. Della Valle ${ }^{6}$,
} P. D'Avanzo ${ }^{1,7}$, A. Grazian ${ }^{2}$, V. Testa ${ }^{2}$, S. Campana ${ }^{1}$, S. Covino ${ }^{1}$, F. Fiore ${ }^{2}$, L. Stella ${ }^{2}$, A. J. Castro-Tirado ${ }^{8}$, J. Gorosabel ${ }^{8}$, D. N. Burrows ${ }^{9}$, M. Capalbi ${ }^{10}$, G. Cusumano ${ }^{11}$, M. L. Conciatore ${ }^{2}$, V. D'Elia ${ }^{2}$, P. Filliatre ${ }^{12,13}$, D. Fugazza ${ }^{1}$, N. Gehrels ${ }^{14}$, P. Goldoni ${ }^{12,13}$, D. Guetta ${ }^{2}$, S. Guziy $^{8}$, E. V. Held ${ }^{15}$, K. Hurley ${ }^{16}$, G. L. Israel ${ }^{2}$,

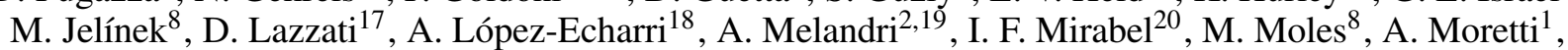
K. O. Mason ${ }^{21}$, J. Nousek ${ }^{9}$, J. Osborne ${ }^{22}$, L. J. Pellizza ${ }^{23}$, R. Perna ${ }^{17}$, S. Piranomonte ${ }^{2}$, L. Piro ${ }^{24}$, A. de Ugarte Postigo ${ }^{8}$, and P. Romano ${ }^{1}$

1 INAF-Osservatorio Astronomico di Brera, via E. Bianchi 46, 23807 Merate (Lc), Italy e-mail: tagliaferri@merate.mi .astro.it

2 INAF-Osservatorio Astronomico di Roma, via di Frascati 33, 00040 Monteporzio Catone (Roma), Italy

3 Università degli studi di Milano-Bicocca, Dipartimento di Fisica, piazza delle Scienze 3, 20126 Milano, Italy

4 Observatori Astronomic, Universitat de Valencia, Aptdo. Correos 22085, Valencia, 46071, Spain

5 International School for Advanced Studies (SISSA-ISAS), via Beirut 2-4, 34014 Trieste, Italy

6 INAF-Osservatorio Astrofisico di Arcetri, largo E. Fermi 5, 50125 Firenze, Italy

7 Dipartimento di Fisica e Matematica, Università dell'Insubria, via Valleggio 11, 22100 Como, Italy

${ }^{8}$ Instítuto de Astrofísica de Andalucía (IAA-CSIC), PO Box 03004, 18080 Granada, Spain

9 Department of Astronomy \& Astrophysics, Pennsylvania State University, State College, PA 16802, USA

${ }_{10}$ ASI Science Data Center, via G. Galilei 5, 00044 Frascati (Roma), Italy

11 INAF-IASF, sezione di Palermo, via U. La Malfa 153, 90146 Palermo, Italy

12 Laboratoire Astroparticule et Cosmologie, UMR 7164, 11 place Marcelin Berthelot, 75231 Paris Cedex 05, France

13 Service d'Astrophysique, DSM/DAPNIA, CEA Saclay, 91911 Gif-sur-Yvette Cedex, France

14 NASA, Goddard Space Flight Center, Greenbelt, MD 20771, USA

15 INAF-Osservatorio Astronomico di Padova, vicolo dell'Osservatorio 5, 35122 Padova, Italy

${ }^{16}$ University of California, Berkeley, Space Sciences Laboratory, Berkeley, CA 94720-7450, USA

17 JILA, University of Colorado, 440 UCB, Boulder CO 80309-0440, USA

18 Instítuto de Astrofísica de Canarias, C/ Vía Láctea s/n, 38200 La Laguna (Tenerife), Spain

19 Università degli Studi di Cagliari, Dipartimento di Fisica, 09042 Monserrato (Ca), Italy

20 European Southern Observatory - Vitacura, Casilla 19001, Santiago 19, Chile

21 MSSL, University College London, Holmbury St. Mary, Dorking, RH5 6NT Surrey, UK

22 X-Ray \& Observational Astronomy Group, Dept. of Physics \& Astronomy, University of Leicester, Leicester LE1 7RH, UK

23 AIM (UMR 7158 CEA/CNRS/Université Paris 7), Service d'Astrophysique, CEA Saclay, 91191 Gif-sur-Yvette, France

24 INAF-IASF, sezione di Roma, via Fosso del Cavaliere 100, 00133 Roma, Italy

Received 26 September 2005 / Accepted 4 October 2005

\section{ABSTRACT}

We present optical and near-infrared observations of the afterglow of the gamma-ray burst GRB 050904. We derive a photometric redshift $z=6.3$, estimated from the presence of the Lyman break falling between the $I$ and $J$ filters. This is by far the most distant GRB known to date. Its isotropic-equivalent energy is $3.4 \times 10^{53} \mathrm{erg}$ in the rest-frame $110-1100 \mathrm{keV}$ energy band. Despite the high redshift, both the prompt and the afterglow emission are not peculiar with respect to other GRBs. We find a break in the $J$-band light curve at $t_{\mathrm{b}}=2.6 \pm 1.0 \mathrm{~d}$ (observer frame). If we assume this is the jet break, we derive a beaming-corrected energy $E_{\gamma} \sim(4 \div 12) \times 10^{51} \mathrm{erg}$. This limit shows that GRB 050904 is consistent with the Amati and Ghirlanda relations. This detection is consistent with the expected number of GRBs at $z>6$ and shows that GRBs are a powerful tool to study the star formation history up to very high redshift.

Key words. cosmology: observations - early Universe - gamma rays: bursts - gamma rays: individual: GRB 050904

\footnotetext{
* Based on observations carried out at ESO telescopes under program Id 075.D-0787. We also used data from Telescopio Nazionale Galileo and Centro Astronómico Hispano Alemán.
} 


\section{Introduction}

Gamma-ray bursts (GRBs) are intense, short pulses of gamma rays, occurring at random positions in the sky. They emit large amounts of energy (up to $\sim 10^{53} \mathrm{erg}$ ) and thus are detectable up to cosmological distances, possibly at $z=10$ and beyond. The discovery of high-redshift GRBs is one of the main goals of the Swift mission (Gehrels et al. 2004) and several ground-based facilities were prepared to be able to detect their counterparts (e.g. Chincarini et al. 2003).

GRBs are accompanied by long-lasting X-ray, optical, and radio counterparts (afterglows). In the first few hours after the explosion, these are much brighter than any known quasar. In particular, optical and near-infrared (NIR) afterglow spectroscopy can easily provide their redshifts and probe the intervening gas along the line of sight. The possible biases affecting the GRB distribution are likely very different from those affecting other classes of objects, such as AGNs, damped Ly $\alpha$ absorbers, and galaxies. Therefore GRBs and their afterglows provide an effective and independent way to probe the highredshift Universe. As a matter of fact, the observed redshift distribution of long-duration GRBs, before the Swift era, peaks at $z \sim 1.6$ and extends up to $z=4.5$ (Andersen et al. 2000). A significant fraction of GRBs has been predicted to occur even at higher redshifts (e.g. Lamb \& Reichart 2000; Gorosabel et al. 2004; Natarajan et al. 2005; Mesinger et al. 2005), during the re-ionization epoch $(6 \lesssim z \lesssim 20$, Gnedin \& Ostriker 1997; Fan et al. 2002; Spergel et al. 2003). Since the progenitors of long-duration GRBs are thought to be short-lived massive stars (Galama et al. 1998; Stanek et al. 2003; Hjorth et al. 2003; Malesani et al. 2004), their detection at large redshifts provides a direct measurement of the star formation rate in the early Universe. Finally, high-redshift GRBs may also serve as useful tools to study the geometry of the Universe (Amati et al. 2002; Ghirlanda et al. 2004).

Our group, the MISTICI ${ }^{1}$ collaboration, has been pursuing follow-up studies of GRB afterglows for several years, concentrating on various aspects of the GRB phenomenon, among them the study of their host galaxies and of foreground DLA systems at high redshift (Fiore et al. 2005; D'Elia et al. 2005). Here we present optical and NIR photometric observations of GRB 050904. This burst was detected by the Burst Alert Telescope (BAT; Barthelmy et al. 2005) onboard Swift on 2005 September 4 at 01:51:44 UT (Cummings et al. 2005). It was a long, bright burst with an observed duration $T_{90}=225 \pm 10 \mathrm{~s}$ (Sakamoto et al. 2005). The $15-150 \mathrm{keV}$ fluence was $(5.4 \pm 0.2) \times 10^{-6} \mathrm{erg} \mathrm{cm}^{-2}$. The BAT spectrum can be described by a hard power law with photon index $\approx 1.2$. Long-lasting flaring activity was detected in the X-ray band up to $50 \mathrm{ks}$ (Cusumano et al. 2005; Watson et al. 2005). A high redshift value for this GRB was first suggested by Haislip et al. (2005a), determined photometrically by us $\left(z=6.1_{-0.12}^{+0.37}\right.$; Antonelli et al. 2005) and then confirmed spectroscopically by Kawai et al. (2005), who measured $z=6.29 \pm 0.01$. A photometric redshift has been derived also by Haislip et al. (2005b) and Price et al. (2005).

\footnotetext{
${ }^{1}$ Multiwavelength Italian Swift Team with International Co-Investigators.
}

Table 1. Observation $\log$ and photometry of the transient source. Errors are at the $1 \sigma$ confidence level, while upper limits are given at $3 \sigma$. Note that the CAFOS and FORS2 I-band filters (which we called $I_{1}$ and $I_{2}$ ) are different (their central wavelengths being $8500 \AA$ and $7680 \AA$, respectively). Since the Lyman $\alpha$ break falls within this band dropout is occuring inside this band, this leads to a significant magnitude difference.

\begin{tabular}{ccrccr}
\hline \hline $\begin{array}{c}\text { Date } \\
(\mathrm{UT})\end{array}$ & Instr. & $\begin{array}{r}t-t_{0} \\
(\mathrm{~d})\end{array}$ & $\begin{array}{c}\text { Exp. } \\
(\mathrm{min})\end{array}$ & Filter & Magnitude \\
\hline 05/09/05 04:47 & ISAAC & 1.125 & 15 & $K_{\mathrm{s}}$ & $18.24 \pm 0.07$ \\
05/09/06 05:23 & ISAAC & 2.146 & 30 & $K_{\mathrm{s}}$ & $19.12 \pm 0.07$ \\
\hline 05/09/05 04:22 & ISAAC & 1.104 & 20 & $H$ & $19.09 \pm 0.07$ \\
05/09/06 04:42 & ISAAC & 2.125 & 30 & $H$ & $19.96 \pm 0.07$ \\
\hline 05/09/05 02:31 & NICS & 1.029 & 50 & $J$ & $19.58 \pm 0.14$ \\
05/09/05 03:55 & ISAAC & 1.087 & 20 & $J$ & $19.92 \pm 0.04$ \\
05/09/06 04:01 & ISAAC & 2.092 & 35 & $J$ & $20.75 \pm 0.07$ \\
05/09/07 04:23 & ISAAC & 3.104 & 60 & $J$ & $21.66 \pm 0.08$ \\
05/09/08 05:44 & ISAAC & 4.162 & 60 & $J$ & $21.91 \pm 0.08$ \\
05/09/09 09:26 & ISAAC & 5.317 & 60 & $J$ & $22.45 \pm 0.19$ \\
05/09/11 06:18 & ISAAC & 7.183 & 102 & $J$ & $>23.2$ \\
\hline 05/09/05 02:25 & WFC & 1.021 & 28 & $z$ & $21.50 \pm 0.25$ \\
05/09/05 07:15 & FORS2 & 1.250 & 90 & $z$ & $21.80 \pm 0.17$ \\
\hline $05 / 09 / 0423: 17$ & CAFOS & 0.892 & 50 & $I_{1}$ & $21.89 \pm 0.20$ \\
05/09/05 01:35 & CAFOS & 0.987 & 50 & $I_{1}$ & $22.03 \pm 0.20$ \\
$05 / 09 / 0503: 38$ & CAFOS & 1.075 & 50 & $I_{1}$ & $22.40 \pm 0.20$ \\
$05 / 09 / 0506: 54$ & FORS2 & 1.208 & 60 & $I_{2}$ & $24.10 \pm 0.18$ \\
\hline $05 / 09 / 0501: 30$ & CAFOS & 0.983 & 90 & $R$ & $>24.1$ \\
\hline $05 / 09 / 0502: 48$ & LAICA & 1.038 & 30 & $V$ & $>24.1$ \\
\hline
\end{tabular}

To date, this is by far the most distant GRB discovered and one of the most distant objects known in the Universe.

\section{Observations and data analysis}

Our observations of the field of GRB 050904 were mostly performed with the $8.2 \mathrm{~m}$ ESO Very Large Telescope (VLT), equipped with the ISAAC and FORS2 instruments (Table 1). Further data were collected with the $2.2 \mathrm{~m}$ and $3.5 \mathrm{~m}$ Calar Alto telescopes (equipped with CAFOS and LAICA, respectively), the $2.5 \mathrm{~m}$ Isaac Newton Telescope (equipped with WFC), and the $3.6 \mathrm{~m}$ Telescopio Nazionale Galileo (equipped with NICS). A NIR counterpart to GRB 050904 was first reported by Haislip et al. (2005a). In our first images, taken on 2005 Sep. 4/5, this object was not present. A pointlike source was however clearly visible in the $I z J H K_{\mathrm{s}}$ bands, at the edge of the XRT error box (Mineo et al. 2005). Its coordinates were $\alpha_{\mathrm{J} 2000}=$ $00^{\mathrm{h}} 54^{\mathrm{m}} 50.83, \delta_{\mathrm{J} 2000}=+14^{\circ} 05^{\prime} 10^{\prime}{ }^{\prime} 0$. We suggested this source to be the afterglow of GRB 050904 (D'Avanzo et al. 2005; see also Fig. 1). Nysewander et al. (2005) subsequently provided revised coordinates for the object of Haislip et al. (2005a), showing it was coincident with our candidate. The afterglow was also detected by the TAROT robotic telescope up to eight minutes after the GRB (Klotz et al. 2005). 


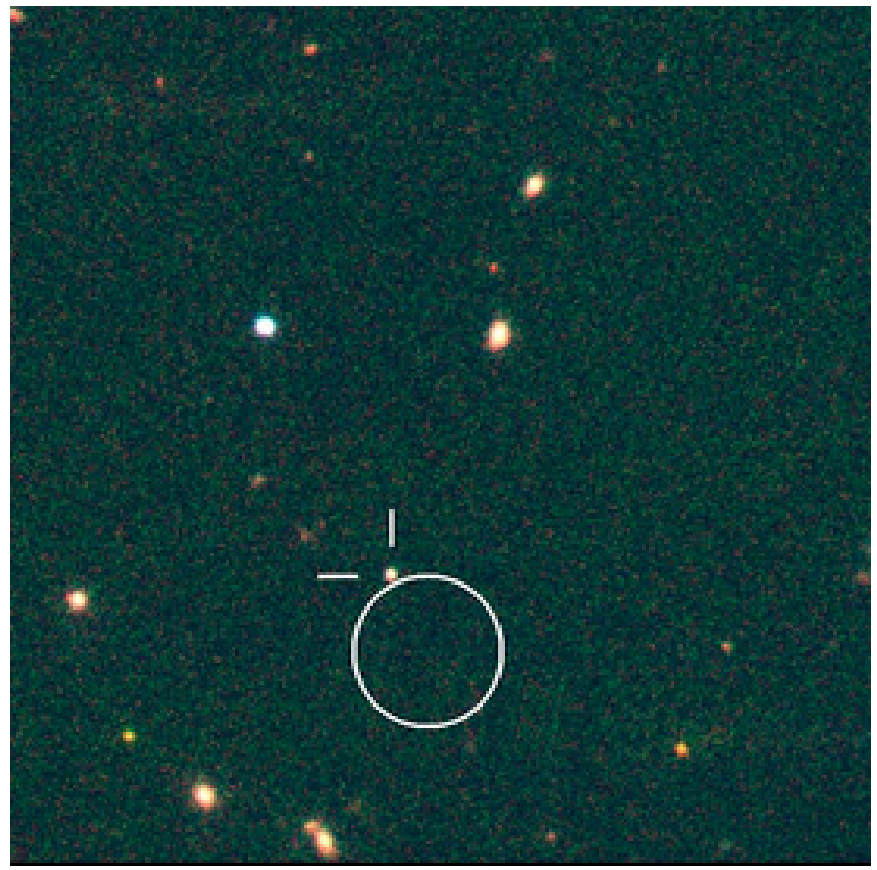

Fig. 1. Three colour-composite image based on our NIR bands $(J, H$, and $K$ ) obtained with VLT+ISAAC on 5 September. The circle represents the 6" XRT afterglow error box. The lines show the position of the optical afterglow. The field is $65^{\prime \prime} \times 65^{\prime \prime}$ wide. North is up, East is left.

Data reduction was carried out following standard procedures. Aperture photometry was performed using SExtractor (Bertin \& Arnouts 1996). Flux calibration was achieved by observing Landolt standard fields ( $I$ band) and against the SDSS ( $z$ band) and 2MASS (NIR bands) surveys.

In Fig. 2 we show our data, together with those by Haislip et al. (2005b). The light curve in the $J$ band, for which we have the best coverage, shows a complex behaviour. Haislip et al. (2005b) have reported a flattening at early times, suggesting that the early emission could be related to a different component, possibly the reverse shock. Our data extend for significantly longer and show a further steepening. Excluding the data at $t<0.4 \mathrm{~d}$, we can model the whole dataset with a smoothly broken power law: $F(t)=2 F_{\mathrm{b}} /\left[\left(t / t_{\mathrm{b}}\right)^{\alpha_{1}}+\left(t / t_{\mathrm{b}}\right)^{\alpha_{2}}\right]$, where $\alpha_{1}$, $\alpha_{2}$ are the early- and late-time slopes, $t_{\mathrm{b}}$ is the break time and $F_{\mathrm{b}}$ is the flux at $t_{\mathrm{b}}$. The fit provides $\alpha_{1}=0.72_{-0.20}^{+0.15}, \alpha_{2}=$ $2.4 \pm 0.4, t_{\mathrm{b}}=2.6 \pm 1.0 \mathrm{~d}$. Errors are at the $1 \sigma$ confidence level throughout the paper.

Figure 3 shows the spectral energy distribution of the afterglow $1.155 \mathrm{~d}$ after the GRB (the epoch around which our measurements cluster). A marked dearth of flux is observed blueward of the $J$ passband, while the $J-H$ and $H-K$ colors are not particularly red. Dust extinction cannot produce such a sharp cutoff, so that a redshifted Lyman dropout must be invoked (due to the strong absorption by neutral hydrogen at wavelengths shorter than $\operatorname{Ly} \alpha$ ). The redshift of GRB 050904 was computed using two different programs. The first is a code based on a widely used technique to derive photometric redshifts of galaxies and quasars (e.g. Fontana et al. 2000), modified to take into account the afterglow properties. In this case we computed a

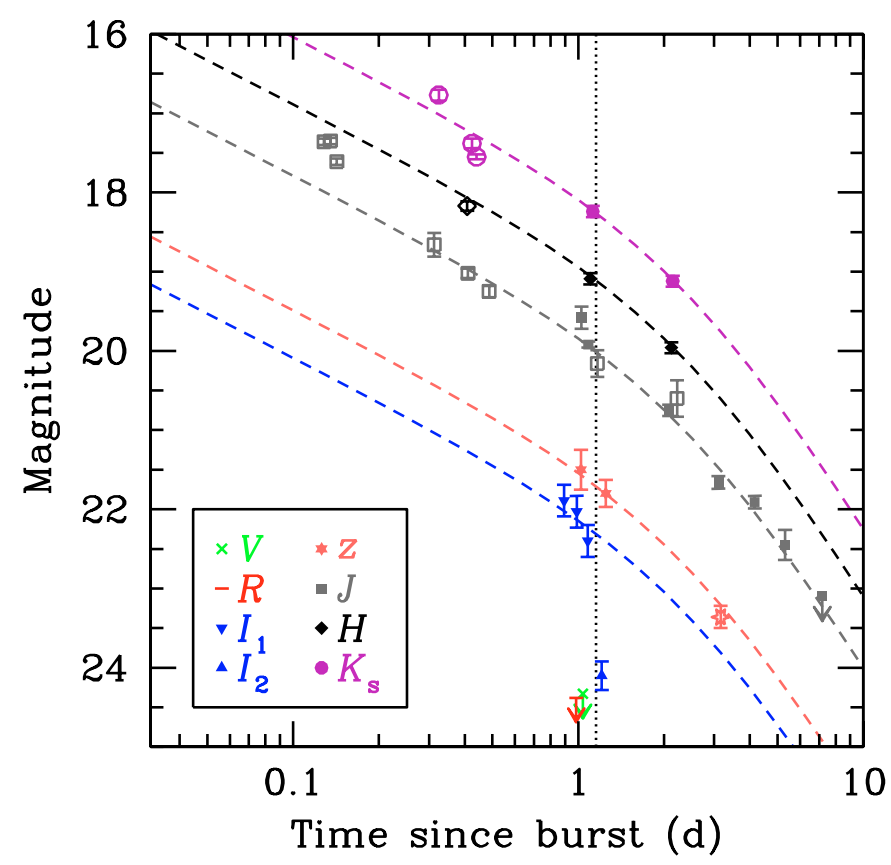

Fig. 2. Light curve of the afterglow of GRB 050904, together with the best fit (dashed lines) computed in the $J$ band. The same fit is reported for the other bands. Open symbols represent data from Haislip et al. (2005b). The vertical dotted line marks the epoch at which we computed the spectral energy distribution (Fig. 3).

library of synthetic GRB afterglow spectra at arbitrary redshifts, modeled with power laws $\left(F_{v} \propto v^{-\beta}\right)$. Reporting all our photometric measurements at a common epoch (adopting the observed decay law), the best fit gives $z=6.30 \pm 0.07$ and $\beta=$ $1.25 \pm 0.25$. The second is the $z-p h-R E M$ code (Fernández-Soto 2004), specifically designed for GRB afterglows and based on the work by Fernández-Soto et al. (1999). This code assumes that the temporal and spectral dependence of the afterglow flux follows a power law $\left(F_{v} \propto t^{-\alpha} v^{-\beta}\right)$. In this case, also including the UVOT optical limits (Cucchiara et al. 2005), the best fit provided $z=6.3 \pm 0.1$ and $\beta=1.2 \pm 0.3$. Both codes use a $\chi^{2}$-minimisation technique to find the best-fitting spectral template to the observed colors. We note that the spectral index is significantly redder than that reported by Price et al. (2005), which was however measured at an earlier epoch.

\section{Discussion}

Despite its high redshift, the optical afterglow of GRB 050904 is not peculiar with respect to other GRBs. For example, its $R$-band spectral luminosity extrapolated at $t=12 \mathrm{~h}$ (rest-frame time) is ${ }^{2} L_{v} \sim 10^{31} \mathrm{erg} \mathrm{s}^{-1} \mathrm{~Hz}^{-1}$, consistent with the values typically observed for previous GRBs (Nardini et al. 2005; Liang \& Zhang 2005). Its spectral and temporal indices are also typical among GRB afterglows. In particular, the afterglow colours leave small room for dust extinction, if any (note that we are observing the rest-frame ultraviolet band). The precise gamma-ray energy budget of this burst is not known, since

\footnotetext{
${ }^{2}$ Assuming a cosmology with $\Omega_{\mathrm{m}}=0.27, \Omega_{\Lambda}=0.73$, and $h_{0}=$ 0.71 .
} 


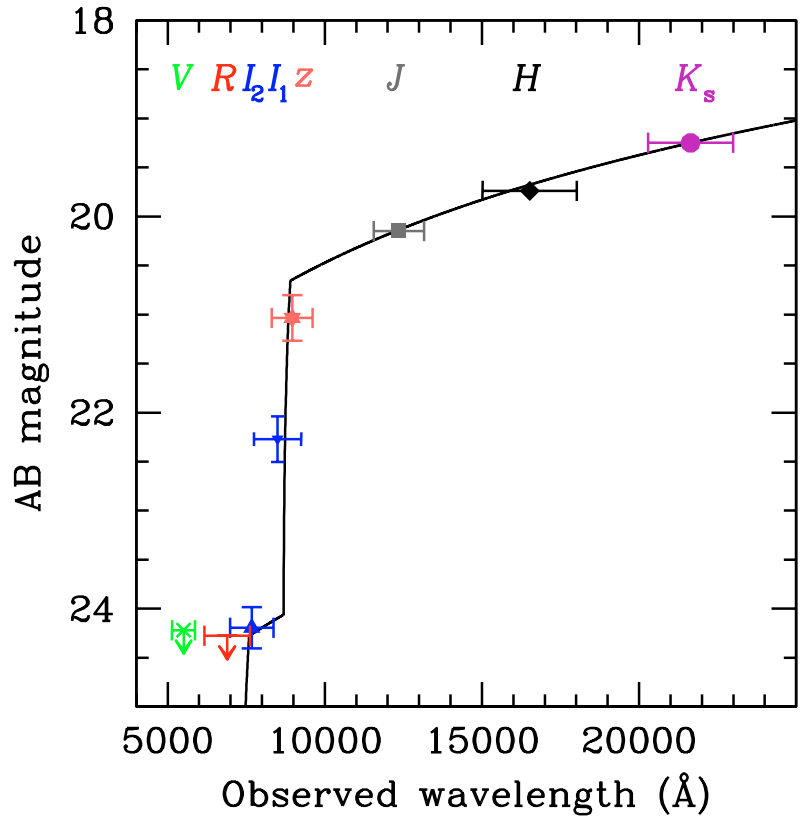

Fig. 3. Photometric spectral energy distribution of the afterglow. Magnitudes were reported at a common epoch (1.155 d after the GRB) using the measured decay law. The solid line shows the best fit model for the afterglow. Data are corrected for Galactic extinction $\left(A_{\mathrm{V}}=0.21 \mathrm{mag}\right)$.

the observed peak spectral energy lies outside the sensitivity BAT range. Cusumano et al. (2005) infer that the isotropicequivalent gamma-ray budget $E_{\gamma \text {,iso }}$ was between $6.6 \times 10^{53}$ and $3.2 \times 10^{54} \mathrm{erg}$, depending on the bolometric correction ( $E_{\gamma \text {,iso }} \simeq 6 \times 10^{52} \mathrm{erg}$ in the $20-100 \mathrm{keV}$ rest-frame band). The steepening in the light curve may be due to a jet break. In this case, adopting the standard formalism (Sari et al. 1999), we can infer a jet half-opening angle $\vartheta_{\text {jet }} \sim 3^{\circ}$, assuming a radiative efficiency $\eta=20 \%$ and a circumburst medium density $n=3 \mathrm{~cm}^{-3}$ (as in Ghirlanda et al. 2004). In this case the beaming-corrected energy would be $E_{\gamma} \sim(4 \div 12) \times 10^{51} \mathrm{erg}$. For this burst the rest-frame peak energy is constrained to be $E_{\mathrm{p}} \gtrsim 1100 \mathrm{keV}$. In the planes $E_{\mathrm{p}}$ vs. $E_{\gamma \text {,iso }}$ and $E_{\mathrm{p}}$ vs. $E_{\gamma}$, the derived limits show that GRB 050904 is consistent with both the Amati and Ghirlanda relations (Amati et al. 2002; Ghirlanda et al. 2004).

The optical/NIR afterglows of GRBs detected by Swift are dimmer than those of other missions, on the average by $2.5 \mathrm{mag}$ at $t=12 \mathrm{~h}$ (Berger et al. 2005). More than one third have no optical counterpart, despite having a well-localized X-ray afterglow. For a good fraction of these bursts, this could be due either to a high redshift and/or to dust extinction (e.g. Zhang \& Mészáros 2004 and references therein; see also Filliatre et al. 2005). In about 10 months of operations, the number of Swift long-duration GRBs with measured redshift has been measured is 16, while the number of non-Swift GRBs with redshift is 43 (obtained over a time period of 7 years). The redshift distribution of the pre-Swift bursts has a peak at $z \sim 1.6$, extending to higher values (the record was $z=4.50$; Andersen et al. 2000). Although the number is still small, the 16 Swift long-duration GRBs with measured redshift have a statistically higher redshift, with an average value of 2.8 (with one object at $z>6$, two with $4<z<6$, three with $3<z<4$ and four with $2<z<3$, e.g. Jakobsson et al. 2005). It is very likely that some of the Swift bursts without optical counterpart are also at high redshift. Moreover, the number of bursts for which it is possible to perform low- and high-resolution spectroscopic studies at moderate redshift $(z>2)$ is rapidly increasing (e.g. D'Elia et al. 2005; Chen et al. 2005).

Finally we note that a GRB survey is much more efficent in finding high-redshift objects than those targetting other sources. For instance only seven quasars out of $\sim 76000$ (selected from a sample of $\sim 850000$ sources for which spectra were acquired, over a $4783 \mathrm{deg}^{2}$ region of the sky) were detected in the Sloan Digital Sky Survey ${ }^{3}$ at $z>6$. For comparison, Swift is monitoring about 1.4 sr of the sky at any time, and it has been able to catch one event at $z>6$, out of a sample of 16 with measured redshift, selected from a sample of $\sim 60 \mathrm{GRBs}$.

\section{Conclusions}

We observed the afterglow of GRB 050904 in the VRIzJHK filters. We derived a photometric redshift $z=6.3 \pm 0.1$ using two independent techniques. This redshift has been estimated by the Lyman break falling between the $I$ and $J$ filters, therefore it is very robust and has been confirmed spectroscopically by Kawai et al. (2005), who measured $z=6.29 \pm 0.01$.

The high redshift of GRB 050904 opens the question of whether its progenitor was a Pop III star (see, for example, Woosley \& Heger 2004; Scannapieco et al. 2005). In this case, however, it would be difficult for the star to lose its envelope. Wind-driven mass loss depends upon metallicity, and it scales as $\dot{M} \propto Z^{0.5 \div 0.9}$ (e.g. Nugis \& Lamers 2000; Kudritzki 2002; Vink \& de Koter 2005). The discovery of GRB 050904, possibly in a low metallicity environment like the one characterizing the early Universe, hints at the need for a close companion that can efficiently strip off the envelope of the progenitor. However, the efficiency of producing GRBs by Pop III stars is currently unknown and may be very low (e.g. Bromm \& Loeb 2005).

A more convincing alternative is the possibility that the progenitor of GRB 050904 was a massive, non-pristine star. This implies that star formation was already active at $z \gtrsim 6$, and that metal enrichment had already started. This finding is consistent with recent measurements of the comoving luminosity density of star-forming galaxies (Giavalisco et al. 2004), which show that the star formation rate varies only slowly with redshift over the range $2<z<6$ (see also Le Fèvre et al. 2005). These results are nevertheless still subject to important - and not well known - corrections due to incompleteness, dust absorption, and systematic effects. GRBs are not affected by dust absorption, and are thus in principle very effective in tracing star formation even in this high redshift range.

Swift has been able to catch one GRB at $z \sim 6$, out of a sample of 16 events for which the redshifts have been measured. By using the whole sample of about 60 GRBs discovered by Swift, we find, as a simple application of Poisson statistics in the small-number regime (Gehrels 1986), that $4_{-3}^{+9}$ events are

\footnotetext{
${ }^{3}$ http://www.sdss.org/dr4/
} 
expected to be discovered at $z>6$. A fraction of about 4/60 $7 \%$, as we have derived on empirical grounds, is consistent with the theoretical estimate recently provided by Bromm \& Loeb (2005), who predicted that about $10 \%$ of the events discovered by Swift are at $z>6$. Once we will be able to measure a significant number of high GRB redshifts, we will be in a position to sample the star formation rate of the Universe within the first Gyr from the Big Bang.

Acknowledgements. This research was supported at OAB and OAR by ASI grant I/R/039/04. We thank A. Fontana for allowing us to use his photometric redshift code. We acknowledge the excellent support of the ESO, TNG and CAHA staff. We also thank the anonymous referee for her/his prompt reply and for useful comments.

\section{References}

Amati, L., Frontera, F., Tavani, M., et al. 2002, A\&A, 390, 81

Andersen, M. I., Hjorth, J., Pedersen, H., et al. 2000, A\&A, 364, L54 Antonelli, L. A., Grazian, A., D'Avanzo, P., et al. 2005, GCN 3924

Barthelmy, S., Barbier, L. M., Cummings, J. R., et al. 2005, Space Sci. Rev., in press [arXiv: astro-ph/0507410]

Berger, E., Kulkarni, S. R., Fox, D. B., et al. 2005, ApJ, submitted [arXiv:astro-ph/0505107]

Bertin, E., \& Arnouts, S. 1996, A\&AS, 117, 393

Bromm, V., \& Loeb, A. 2005, ApJ, submitted [arXiv:astro-ph/0509303]

Chen, H.-W., Prochaska, J. X., Bloom, J. S., \& Thompson, I. B. 2005, ApJ, submitted [arXiv: astro-ph/0508270]

Chincarini, G., Zerbi, F. M., Antonelli, L. A., et al. 2003, The Messenger, 113, 40

Cucchiara, A., Cummings, J., Holland, S. T., et al. 2005, GCN 3923

Cummings, J., Angelini, L., Barthelmy, S., et al. 2005, GCN 3910

Cusumano, G., Mangano, V., Chincarini, G., et al. 2005, Nature, submitted [arXiv: astro-ph/0509737]

D’Avanzo, P., Antonelli, L. A., Covino, S., et al. 2005, GCN 3921

D'Elia, V., Melandri, A., Fiore, F., et al. 2005, GCN 3746

Fan, X., Narayanan, V. K., Strauss, M. A., et al. 2002, AJ, 123, 1247

Fernández-Soto, A., Lanzetta, K. M., \& Yahil, A. 1999, ApJ, 513, 34

Fernández-Soto, A. 2004, in Multiwavelength Cosmology, ed. M. Plionis

Filliatre, P., Covino, S., D'Avanzo, P., et al. 2005, A\&A, submitted

Fiore, F., D’Elia, V., Lazzati, D., et al. 2005, ApJ, 624, 853

Fontana, A., D'Odorico, S., Poli, F., et al. 2000, AJ, 120, 2206

Galama, T. J., Vreeswijk, P. M., van Paradijs, J., et al. 1998, Nature, 395,670
Gehrels, N. 1986, ApJ, 303, 336

Gehrels, N., Chincarini, G., Giommi, P., et al. 2004, ApJ, 611, 1005

Ghirlanda, G., Ghisellini, G., \& Lazzati, D. 2004, ApJ, 616, 331

Giavalisco, M., Dickinson, M., Ferguson, H., et al. 2004, ApJ, 600, L103

Gnedin, N. Y., \& Ostriker, J. P. 1997, ApJ, 486, 581

Gorosabel, J., Lund, N., Brandt, S., Westergaard, N. J., \& Castro Cerón, J. M. 2004, A\&A, 426, 87

Haislip, J. B., Reichart, D. E., Cypriano, E., et al. 2005a, GCN 3913

Haislip, J. B., Nysewander, M. C., \& Reichart, D. E., et al. 2005b, Nature, submitted [arXiv: astro-ph/0509660]

Hjorth, J., Sollerman, J., Moller, P., et al. 2003, Nature, 423, 847

Jakobsson, P., Levan, A., Fynbo, J. P. U., et al. 2005, A\&A, submitted [arXiv: astro-ph/0509888]

Kawai, N., Yamada, T., Kosugi, G., Hattori, T., \& Aoki, K. 2005, GCN 3937

Klotz, A., Boer, M., \& Atteia, J. L. 2005, GCN 3917

Kudritzki, R. 2002, ApJ, 577, 389

Lamb, D. Q., \& Reichart, D. E. 2000, ApJ, 536, 1

Le Fèvre, O., Paltani, S., Arnouts, S., et al. 2005, Nature, 437, 519

Liang, E., \& Zhang, B. 2005, Nature, submitted

[arXiv: astro-ph/0508510]

Malesani, D., Tagliaferri, G., Chincarini, G., et al. 2004, ApJ, 609, L5

Mesinger, A., Perna, R., \& Haiman, Z. 2005, ApJ, 623, 1

Mineo, T., Mangano, V., La Parola, V., et al. 2005, GCN 3920

Nardini, M., Ghisellini, G., Ghirlanda, G., et al. 2005, A\&A, submitted [arXiv: astro-ph/0508447]

Natarajan, P., Albanna, B., Hjorth, J., et al. 2005, MNRAS, in press [arXiv: astro-ph/0505496]

Nugis, T., \& Lamers, H. 2000, A\&A, 360, 227

Nysewander, M., Reichart, D., \& Haislip, J. 2005, GCN 3922

Price, P. A., Cowie, L. L., Minezaki, T., et al. 2005, ApJ, submitted [arXiv:astro-ph/0509697]

Sakamoto, T., Barbier, L., Barthelmy, S., et al. 2005, GCN 3938

Sari, R., Piran, T., \& Halpern, J. 1999, ApJ, 519, L17

Scannapieco, E., Madau, P., Woosley, S., Heger, A., \& Ferrara, A. 2005, ApJ, in press [arXiv:astro-ph/0507182]

Spergel, D. N., Verde, L., Peiris, H. V., et al. 2003, ApJS, 148, 175

Stanek, K. Z., Matheson, T., Garnavich, P. M., et al. 2003, ApJ, 591, L17

Vink, J., \& de Koter, A. 2005, A\&A, 442, 587

Watson, D., Reeves, J. N., Hjorth, J., et al. 2005, ApJ, submitted [arXiv: astro-ph/0509640]

Woosley, S., \& Heger, A. 2004, IAU Symp., 215, 601

Zhang, B., \& Mészáros, P. 2004, Int. Journ. Mod. Phys. A, 19, 2385 